\title{
Highly uniform micro-cavity arrays in flexible elastomer film
}

\author{
Shahab Shojaei-Zadeh, Shaun R. Swanson and Shelley L. Anna* \\ Received 13th May 2008, Accepted 16th December 2008 \\ First published as an Advance Article on the web 19th January 2009 \\ DOI: $10.1039 / \mathrm{b808109c}$
}

\begin{abstract}
Uniform hexagonal arrays of spherical micro-cavities are created in an elastomer film in a one-step process. The cavities are the imprints of water droplets condensed from saturated vapor in a heated closed container. The saturated vapor arises from evaporation of water from a reservoir within the container. Competition between droplet growth and curing of the elastomer film determines the structure of the resulting pattern.
\end{abstract}

Materials containing highly ordered microscale structure exhibit unique and useful properties. When the periodicity of the structure is comparable to the wavelength of light, materials can act as optical stopbands or photonic switches. ${ }^{1}$ Microscale structure can provide templates for tissue engineering ${ }^{2}$ or picoliter beakers. ${ }^{3}$ The significant potential for application of ordered materials has led to many fabrication methods, from top down lithography and microcontact printing $^{4}$ to 'bottom-up' natural and driven assembly. For example, evaporation of a suspension on a substrate drives assembly of hexagonal close-packed layers of colloidal particles. ${ }^{5}$ Spin coating ${ }^{6}$ and colloidal epitaxy ${ }^{7}$ can also control order. Convection cells yield single layer periodic patterns, ${ }^{8}$ while 'breath figure templated assembly' can yield multi-layer bubble arrays from water droplets condensing in a humid gas stream onto the surface of a volatile polymer solution. ${ }^{9-11}$ These methods offer many patterning options for a wide range of materials. A common disadvantage is that films containing the desired microstructure are often not mechanically stable, requiring additional fabrication steps such as annealing and the use of sacrificial materials. ${ }^{3}$

The present paper describes a one-step process in which a layer of spherical cavities is created within a mechanically stable elastomer film of cross-linked poly(dimethylsiloxane) (PDMS). The optical micrograph in Fig. 1(a) shows that the spherical objects are highly uniform, less than $10 \mu \mathrm{m}$ in radius, and well ordered in a hexagonal close-packed array. The SEM micrographs in Fig. 1(b) and (c) show that the spheres are hollow cavities, and that these cavities reside in a single layer at the top surface of the film. The bright spots in the optical micrograph at the center of each cavity coincide with tiny holes that perforate the thin films at the top surface of each cavity, shown in the SEM micrographs. The thin films between the cavities are also perforated.

The process used to generate the micro-cavity arrays is illustrated in Fig. 2. At room temperature $T_{\mathrm{i}}$, a clean glass jar is partially filled with volume $V_{\mathrm{w}}$ of deionized water. Volume $V_{\mathrm{p}}$ of liquid PDMS is slowly dispensed onto the water surface to form a thin film supported by the reservoir. The PDMS (Dow Sylgard 184) has been thoroughly mixed with a non-volatile cross-linking agent and degassed. The film

Department of Mechanical Engineering, Carnegie Mellon University, Pittsburgh PA 15213. E-mail: sanna@cmu.edu does not initially reach the walls of the jar, leaving water surface exposed. Immediately after dispensing PDMS, the container is tightly closed and placed in a hot convection oven at temperature $T_{\mathrm{f}}$ for one hour to cure the elastomer film. The cured film is removed from the water surface, dried in a nitrogen stream, and observed with a microscope. The size, uniformity, surface coverage, and degree of ordering of the micro-cavities depend on the PDMS and water volumes, the concentration of crosslinking agent, and the initial and final temperatures. For purposes of the present study we hold the container geometry fixed, $R=1.4 \mathrm{~cm}, H=3 \mathrm{~cm}$, and we do not vary the fluids.

The current experiment configuration does not permit direct monitoring of micro-cavity formation. However, we obtain indirect clues to the controlling mechanisms in three separate experiments: (1) we omit the water reservoir, (2) we allow the liquid PDMS film to spread completely over the reservoir surface prior to heating, and (3) we remove the lid to the container during heating and curing. In all three cases, the cured elastomer film is transparent and contains no cavities. In addition, we conduct experiments in which the uncured PDMS is placed in a separate dish inside the closed vapor space, with its top surface exposed to the vapor. If the dish containing PDMS is

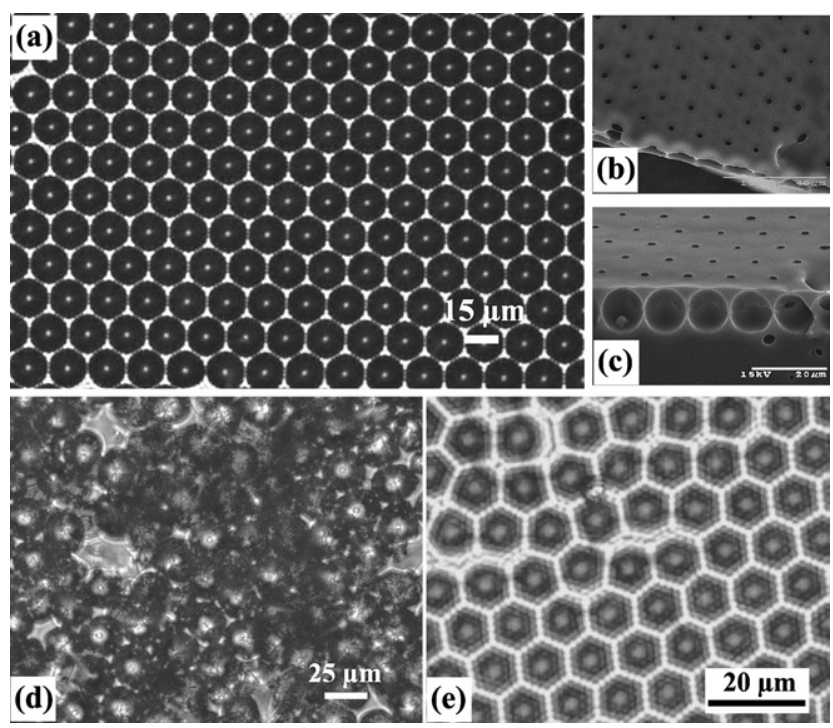

Fig. 1 (a) An optical micrograph of micro-cavity array formed in PDMS with $2: 1$ crosslinker ratio. The radius of the spherical cavities shown is $7.6 \pm 0.3 \mu \mathrm{m}$. (b) A top view SEM micrograph shows 1 micron holes perforating the top layer of PDMS above each cavity (scale bar $40 \mu \mathrm{m}$ ). (c) An angled view SEM micrograph shows a single layer of hollow spheres (scale bar $20 \mu \mathrm{m}$ ). Films between cavities are perforated by 1 micron holes. (d) Disordered structures arise in some conditions. (e) Honeycomb structures form from highly packed droplets. 
surrounded by vapor and not in thermal contact with the water reservoir, cavities are not present in the final film. If the container floats on the surface of the water reservoir during curing, providing thermal contact with the PDMS film, cavities form as shown in Fig. 1.

These observations taken together lead to the hypothesis that the cavities are the imprints of water droplets that have condensed onto the PDMS surface subsequent to evaporation from the exposed reservoir surface. During evaporation, warm humid air collects in the space above the reservoir and is unable to escape the container. At the same time, the uncured PDMS film is in thermal contact with the water reservoir, which reaches thermal equilibrium much more slowly than the rest of the system. Since the PDMS surface temperature is held at a lower temperature than the vapor saturation temperature at a given time, condensation of water vapor onto the surface is favorable. Condensed droplets can sink into the PDMS film since PDMS is slightly less dense than water, $\rho_{\mathrm{p}}=923 \mathrm{~kg} \mathrm{~m}^{-3}$. However, droplets are expected to remain spherical and settle very slowly since gravity is very small compared with surface tension, as reflected in the Bond number $B o \equiv\left(\rho_{\mathrm{w}}-\rho_{\mathrm{p}}\right) g r_{\mathrm{c}}{ }^{2} / \gamma_{\mathrm{wp}} \approx 1 \times 10^{-6}$, where $\rho_{\mathrm{w}}=995 \mathrm{~kg} \mathrm{~m}^{-3}$ is the water density, $r_{\mathrm{c}}$ is the cavity radius, and $\gamma_{\mathrm{wp}}=$ $34.8 \mathrm{mN} \mathrm{m}^{-1}$ is the interfacial tension at the water-PDMS surface.

Our process is similar in some ways to the process known as 'breath figure templated assembly' for forming ordered macroporous films, ${ }^{9-11}$ in which warm humid air flows over the surface of a polymer film containing volatile solvent. As the volatile solvent evaporates, evaporative cooling promotes condensation of water droplets on the polymer surface, and cavities result when the water droplets sink into the film and leave behind an imprint upon film drying. Despite these similarities with the breath figure templated assembly process, it is important to note that we cannot achieve patterns in PDMS films via the same process. Flowing warm humid air over the PDMS surface results in a transparent film with few cavities. In addition, as discussed below, the evaporation and condensation processes occur at rates

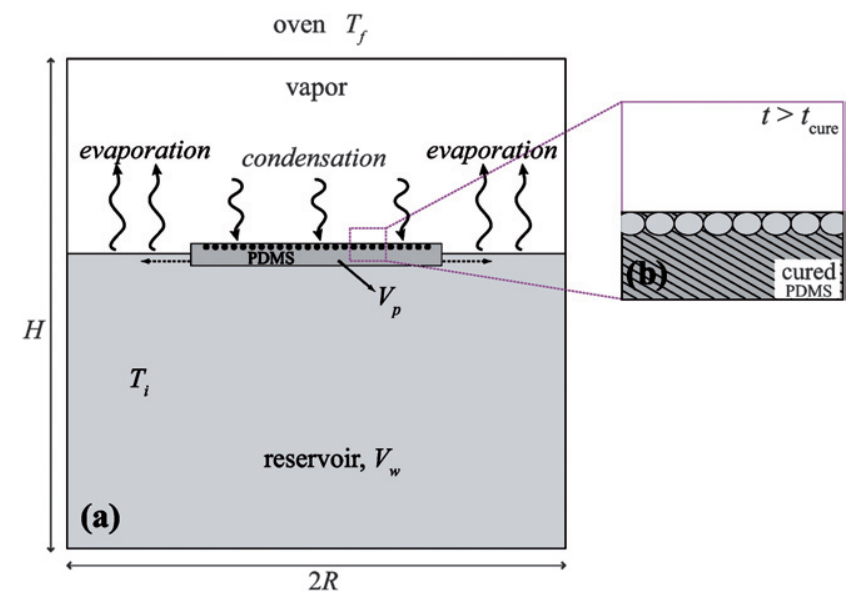

Fig. 2 A schematic diagram of micro-cavity formation. (a) Liquid silicone partially covers the surface of a water reservoir. The closed cylindrical container, height $H$ and radius $R$, is heated in a convection oven. Water evaporates from exposed reservoir surface and condenses onto the curing film. (b) Condensing droplets cluster into hexagonal structures while the viscous films separating them prevent coalescence. The cured elastomer mechanically stabilizes the structure. Water evaporates, leaving behind a hollow imprint of the droplets. comparable to that of the curing process for the PDMS film. Thus, film curing arrests pattern formation, controlling the number of layers and the degree of ordering of the cavities within the layers.

Estimates of heat transfer rates within the system shown schematically in Fig. 2 indicate that the rate of heating of the water reservoir is a controlling parameter in pattern formation. The characteristic timescale for heat conduction within a material is given by $\tau_{\mathrm{c}}$ $\approx L^{2} / \alpha$, where $L$ is the distance over which conduction occurs and $\alpha$ is the thermal diffusivity. The thermal diffusivities of water $(\alpha=$ $\left.1.5 \times 10^{-7} \mathrm{~m}^{2} \mathrm{~s}^{-1}\right)$, PDMS $\left(\alpha=1.48 \times 10^{-7} \mathrm{~m}^{2} \mathrm{~s}^{-1}\right)$, and glass $(\alpha=$ $\left.3.4 \times 10^{-7} \mathrm{~m}^{2} \mathrm{~s}^{-1}\right)$ are comparable, while that of air is significantly larger $\left(\alpha=2.2 \times 10^{-5} \mathrm{~m}^{2} \mathrm{~s}^{-1}\right)$. Considering the characteristic lengthscales for the air and water volumes $(L \approx R \approx 1.4 \mathrm{~cm})$, the glass jar $(L \approx$ thickness of jar wall $\approx 1 \mathrm{~mm})$, and the PDMS film ( $L \approx$ thickness of poured film $\approx 1 \mathrm{~mm}$ ), we estimate the timescales for heat conduction in each component. The characteristic timescale for conduction through the walls of the glass jar $(\tau \approx 3 \mathrm{~s})$, across the PDMS film $(\tau \approx 7 \mathrm{~s})$ and across the vapor space $(\tau \approx 9 \mathrm{~s})$ are comparable to each other and significantly faster than that of the water reservoir $(\tau \approx 1400 \mathrm{~s}$ ). This estimate is consistent with direct measurements of the temperature in the water reservoir. A thermocouple placed within the reservoir shows that the water temperature approaches the oven temperature after more than 80 min (Fig. 3). Assuming conduction is the primary heat transfer mechanism in the reservoir, a lumped heat capacity model ${ }^{12}$ predicts an exponential temperature rise with time $t$ given by

$$
\left(T_{\mathrm{w}}-T_{\mathrm{f}}\right)=\left(T_{\mathrm{i}}-T_{\mathrm{f}}\right) \exp [-(h A / \rho c V) t]
$$

where $T_{\mathrm{w}}$ is the temperature of the water reservoir, $h$ is a heat transfer coefficient, $A$ is the cross-sectional area of the reservoir, $\rho$ is the water density, $c$ is the heat capacity of water, and $V$ is the volume of the reservoir. The measured exponential temperature rise shown in Fig. 3 yields a fitted heat transfer coefficient of $h=31 \mathrm{~W} \mathrm{~m}^{-2}{ }^{\circ} \mathrm{C}$, which is consistent with empirical values reported in other geometries. ${ }^{12}$ Eqn (1) indicates that the larger the reservoir, the more slowly the water

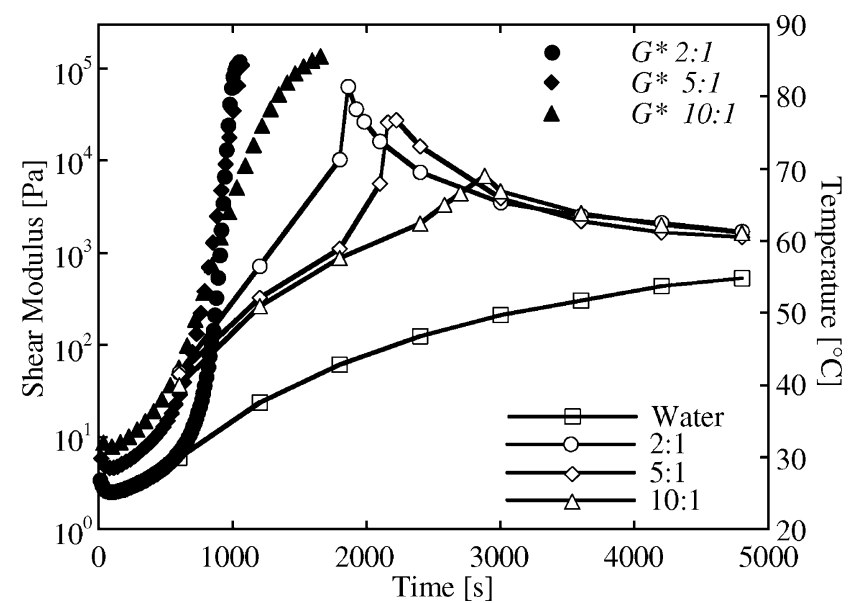

Fig. 3 Shear moduli $G^{*}$ of PDMS films curing at $60^{\circ} \mathrm{C}$ for $2: 1(\bullet), 5: 1$ $(\diamond)$, and $10: 1(\boldsymbol{\Delta})$ crosslinker ratios. As a film cures, $G^{*}$ increases rapidly. Open symbols show the temperature within films curing in a $60{ }^{\circ} \mathrm{C}$ convection oven. The temperature of the water reservoir $(\square)$ rises slowly by comparison, as measured in a $R=1.3 \mathrm{~cm}, H=4 \mathrm{~cm}$ jar. 
temperature equilibrates, and the larger the instantaneous temperature difference between the reservoir and the vapor.

Evaporation from the surface of a volatile liquid generally occurs when the surface is warmer than the vapor directly above it, while condensation occurs at cooler surfaces. Evaporation and condensation rates can be estimated empirically using heat transfer coefficients $h_{\mathrm{e}}$ and $h_{\mathrm{c}}$ respectively, where the mass rate of evaporation of water $\dot{m}_{\mathrm{e}}$ from the surface of the slowly heating water reservoir is proportional to the exposed surface area $A_{\mathrm{e}}$ and the temperature difference, $\dot{m}_{\mathrm{e}}=$ $h_{\mathrm{e}} A_{\mathrm{e}}\left(T_{\mathrm{f}}-T_{\mathrm{w}}\right) / h_{\mathrm{fg}}$, where $h_{\mathrm{fg}}$ is the enthalpy of vaporization. Similarly, the mass flux associated with condensation is given by $m \dot{t}_{\mathrm{c}}=h_{\mathrm{c}} A_{\mathrm{p}}\left(T_{\mathrm{p}}\right.$ $\left.-T_{\mathrm{f}}\right) / h_{\mathrm{fg}}$, where $A_{\mathrm{p}}$ is the surface area of the PDMS film and $T_{\mathrm{p}} \approx T_{\mathrm{w}}$ is the temperature of the PDMS film. The heat transfer coefficients depend on the geometry of the system, fluid properties, and the temperature difference. In addition, condensation is particularly difficult to model since heat dissipation in the condensing film must be taken into account. If condensation occurs in a dropwise manner, surface tension and nonuniform heat transfer effects can also be significant. ${ }^{13,14}$ Combined with eqn (1), it is clear that the evaporation and condensation rates change with time but that both processes will occur over a long time period within our system. In general, larger temperature differences lead to larger rates of evaporation and condensation, ${ }^{12}$ which we expect to occur when the water reservoir is larger.

Although condensation rates are difficult to estimate, Fig. 3 shows that PDMS cures in a timescale comparable to that at which the temperature of the water reservoir equilibrates. The figure shows the cure kinetics of PDMS as a function of crosslinker concentration, where closed symbols represent the complex modulus $G^{*}$ measured at a frequency of $1 \mathrm{~Hz}$ during curing at $60^{\circ} \mathrm{C}$ in a rotational rheometer. The modulus is initially low and dominated by the viscous component, then rises rapidly after about $900 \mathrm{~s}$ to a large elastic plateau, signifying complete curing. Larger crosslinker concentrations result in lower initial viscosities, later onset of curing, and faster curing once started. In addition, the open symbols in Fig. 3 indicate the temperature evolution in curing PDMS films, where the large temperature spike indicates release of heat during exothermic curing. The data indicates that PDMS curing is complete within 15-30 min. Since the water reservoir has not reached equilibrium within this timeframe, evaporation and condensation are likely to occur during the entire curing process, and thus we expect the cure kinetics to be the limiting factor in the pattern formation process. Furthermore, we note that droplets condensing on a viscous oil surface have been observed to grow with a power law in time, $R \propto t^{1 / 3}$, consistent with a constant rate of mass flux, and to become visible at $1-2 \mu \mathrm{m}$, about $1 \mathrm{~s}$ after flowing humid gas over the substrate. ${ }^{15,16}$ Although the exact rates of growth will vary in our process, this information suggests that condensing droplets will reach the size of the cavities shown in Fig. 1 several minutes after heating begins. Thus, the comparable timescales for droplet condensation, growth, and film curing are consistent with the appearance of a single layer of cavities within the film.

For dense, well-ordered cavity arrays, the cavity size is an indication of the competition between the rate of condensation and the rate of film curing. Based on the above analysis, we expect that the water reservoir volume, the initial temperature and the oven temperature will be key parameters controlling the cavity size. For fixed PDMS volume $V_{\mathrm{p}}=1 \mathrm{ml}$ and a $2: 1$ crosslinker ratio, we systematically vary $V_{\mathrm{w}}, T_{\mathrm{i}}$, and $T_{\mathrm{f}}$. For a given set of temperatures, the cavity radius generally increases with water volume, as shown in Fig. 4. This trend is consistent

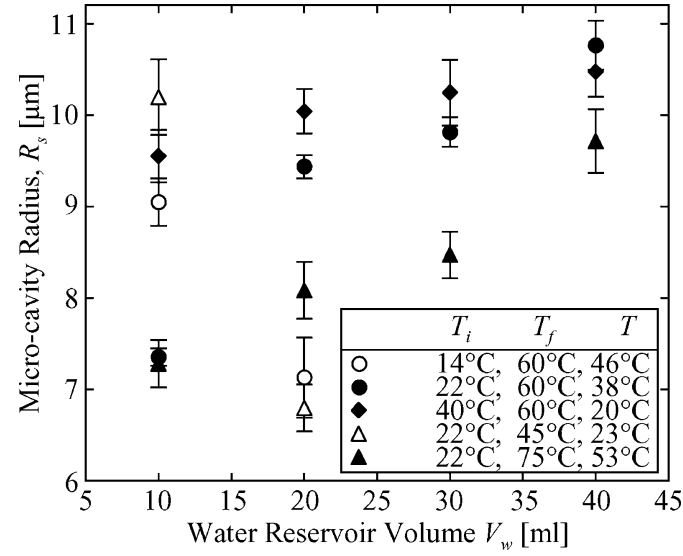

Fig. 4 Measured micro-cavity radius as a function of reservoir volume for five temperature pairs $\left(T_{\mathrm{i}}, T_{\mathrm{f}}\right)$.

with our expectation that evaporation and condensation rates increase with increasing water volume. Fig. 4 also shows that the variation of pore radius depends on the two temperatures independently and not simply on the temperature difference $\Delta T=T_{\mathrm{f}}-T_{\mathrm{i}}$. We presume that this complex temperature dependence is a result of complicated heat transfer occurring during the condensation process. The smallest $\Delta T=$ $20{ }^{\circ} \mathrm{C}$ exhibits the weakest pore size dependence on water volume, a moderate $\Delta T=38^{\circ} \mathrm{C}$ exhibits the fastest rate of increase, and the largest $\Delta T=53^{\circ} \mathrm{C}$ exhibits a moderate rate of increase. In two cases, corresponding to the lowest initial temperature $T_{\mathrm{i}}=14{ }^{\circ} \mathrm{C}$ and the lowest oven temperature $T_{\mathrm{f}}=45^{\circ} \mathrm{C}$, the pore size decreases with increasing water volume and no cavities are observed for $V_{\mathrm{w}}>20 \mathrm{ml}$ in either case. In the latter case, none of the observed patterns are ordered. It is clear that the process is complicated, in part due to complex condensation dynamics. In addition, we note that cure kinetics depend on temperature, further impeding our ability to quantitatively predict the cavity size as a function of the controlling variables.

Although trends in observed cavity size are consistent with the relevant mass transfer rates in the system, it is not understood why the cavity sizes are so uniform. Typical dropwise condensation processes involve coalescence of condensing droplets on the surface, leading to polydispersity in the observed droplet sizes. ${ }^{14}$ Although the highly viscous PDMS between the droplets will drain slowly and inhibit coalescence, we also note that water droplets are rapidly encapsulated within liquid PDMS at isothermal conditions, similar to observations on other polymeric liquids. ${ }^{17}$ Encapsulation is easily understood by considering the interfacial tension values, measured using a du Nouy ring tensiometer: $\gamma_{\mathrm{aw}}=68.2 \mathrm{mN} \mathrm{m}^{-1}, \gamma_{\mathrm{ap}}=18.2 \mathrm{mN} \mathrm{m}^{-1}$, and $\gamma_{\mathrm{wp}}=$ $34.8 \mathrm{mN} \mathrm{m}^{-1}$. Based on these values, the spreading coefficient is large and negative, $S=\gamma_{\mathrm{ap}}-\gamma_{\mathrm{wp}}-\gamma_{\mathrm{aw}}=-84.8 \mathrm{mN} \mathrm{m}^{-1}$, indicating that a sessile droplet is not stable and encapsulation is highly likely. ${ }^{18}$ Although PDMS is slightly permeable to water, we assume that the droplets stop growing once covered with PDMS since permeation is much slower than condensation. ${ }^{19}$ Therefore, if dropwise condensation occurs, an additional mechanism must exist allowing the water droplets to remain uncovered until they have grown to a larger size. One possibility is that significant thermal Marangoni effects stabilize the droplets during condensation. Another possibility is that condensation occurs in a film and is followed by a dewetting transition. ${ }^{20}$ None of these mechanisms can be validated or refuted without additional tools for observing the developing pattern. 
Finally, we note that several system variables impact the degree of ordering of the cavity array. Since the timescales for curing and growth are similar, the competition between the processes determines whether an ordered pattern [Fig. 1(a)] or a disordered pattern [Fig. 1(d)] results. We find that surface coverage is greatest for the smallest reservoir volume $V_{\mathrm{w}}=10 \mathrm{ml}$, where a dense, foam-like honeycomb pattern with significantly deformed and faceted borders is observed as shown in Fig. 1(e). The coverage is denser in the center of the film since it is thicker and cures more slowly, allowing more cavities to form prior to curing.

The degree of ordering increases with the concentration of crosslinking agent. For volumes $V_{\mathrm{p}}=1 \mathrm{ml}$ and $V_{\mathrm{w}}=10 \mathrm{ml}$, a $20: 1$ weight ratio of PDMS to crosslinker yields a transparent film without cavities, while a $10: 1$ ratio results in a disordered pattern similar to Fig. 1(d). A $5: 1$ ratio yields a greater degree of order, and a 2:1 ratio leads to a highly ordered pore pattern similar to Fig. 1(a), in which there are very few defects over a large area $\left(\sim 10 \mathrm{~cm}^{2}\right)$. This makes sense since the viscosity remains low longer (Fig. 3), promoting mobility of the condensed droplets. Droplets condensing on a liquid substrate are known to be significantly more mobile than on a solid substrate, and are observed to cluster into hexagonal arrays due to attractive capillary forces deforming the liquid surface. ${ }^{16}$ Lastly, the volume of dispensed PDMS also influences ordering. For a $2: 1$ crosslinker ratio and $V_{\mathrm{w}}=10 \mathrm{ml}$, a small dispensed volume $V_{\mathrm{p}}=$ $0.5 \mathrm{ml}$ results in a disordered pattern similar to Fig. 1(d). Larger PDMS volumes $V_{\mathrm{p}} \geq 2 \mathrm{ml}$ spread more quickly to cover the reservoir surface and thus result in transparent films containing few or no cavities. An intermediate volume $V_{\mathrm{p}}=1 \mathrm{ml}$ results in a highly ordered pattern similar to Fig. 1(a). We note that neither $V_{\mathrm{p}}$ nor the crosslinker concentration strongly influence the cavity size.

In summary, we have described a process by which water droplets will nucleate and grow on a liquid PDMS film due to condensation from saturated vapor in a closed heated container. Condensation continues as long as a supply of saturated vapor is available. Once a condensing water droplet grows large enough, it is encapsulated within the PDMS and growth stops. The encapsulated droplets cluster into hexagonal arrays until the PDMS film cures and stabilizes the pattern. Clearly, visualization of this complicated process is needed to elucidate some of the underlying mechanisms. However, we have described a simple, robust process by which a uniform, ordered, and mechanically stable macro-porous membrane can form. Developing an understanding of this process will allow greater control over the membrane properties and lend insight into similar physical processes such as the formation of breath figures.

We thank Steve Garoff, Alan McGaughey, Yoed Rabin, Kathleen Stebe, and James Gilchrist for helpful discussions and suggestions. We are grateful for support from the National Science Foundation (NSF CBET-0547432). S. Swanson thanks the Undergraduate Research Office at Carnegie Mellon University for support of his summer research project.

\section{Notes and references}

1 P. Jiang, G. N. Ostojic, R. Narat, D. M. Mittleman and V. L Colvin, Adv. Mater., 2001, 13, 389.

2 A. Mata, C. Boehm, A. J. Fleischman, G. Muschler and S. Roy, J. Biomed. Mater. Res., 2002, 62, 499.

3 B. Erdogan, L. L. Song, J. N. Wilson, J. O. Park, M. Srinivasarao and U. H. F. Bunz, J. Am. Chem. Soc., 2004, 126, 3678.

4 C. Donzel, M. Geissler, A. Bernard, H. Wolf, B. Michel, J. Hilborn and E. Delamarche, Adv. Mater., 2001, 13, 1164.

5 P. Jiang, J. F. Bertone, K. S. Hwang and V. L. Colvin, Chem. Mater., 1999, 11, 2132.

6 M. S. Park, W. Joo and J. K. Kim, Langmuir, 2006, 22, 4594.

7 A. van Blaaderen, R. Ruel and P. Wiltzius, Nature, 1997, 385, 321.

8 M. Q. Li, S. Q. Xu and E. Kumacheva, Macromolecules, 2000, 33, 4972.

9 G. Widawski, M. Rawiso and B. Francois, Nature, 1994, 369, 387.

10 M. Srinivasarao, D. Collings, A. Philips and S. Patel, Science, 2001, 292, 79.

11 U. H. F. Bunz, Adv. Mater., 2006, 18, 973.

12 J. G. Holman, Heat Transfer, McGraw-Hill, New York, 1990.

13 D. Fritter, C. M. Knobler and D. A. Beysens, Phys. Rev. A, 1991, 43, 2858.

14 S. J. Gokhale, J. L. Plawsky and P. C. Wayner, J. Colloid Interface Sci. 2003, 259, 354.

15 D. Beysens and C. M. Knobler, Phys. Rev. Lett., 1986, 57, 1433.

16 C. M. Knobler and D. Beysens, Europhys. Lett., 1988, 6, 707.

17 E. Bormashenko, A. Musin, Y. Bormashenko, G. Whyman, R. Pogreb and O. Gendelman, Macromol. Chem. Phys., 2007, 208, 702 .

18 R. Aveyard and J. H. Clint, J. Chem. Soc., Faraday Trans., 1997, 93, 1397.

19 G. C. Randall and P. S. Doyle, Proc. Natl. Acad. Sci. U. S. A., 2005, 102, 10813.

20 K. Kargupta and A. Sharma, J. Colloid Interface Sci., 2002, 245, 99. 